\title{
NANOTECHNOLOGY-BASED SYSTEMS FOR NUCLEAR RADIATION AND CHEMICAL DETECTION
}

\author{
Kody Varahramyan (PI), Pedro Derosa, Chester Wilson \\ Institute for Micromanufacturing \\ Louisiana Tech University, Ruston, Louisiana 71272
}

\section{EXECUTIVE SUMMARY}

This constitutes the final report of the project on Nanotechnology for Nuclear Nonproliferation Applications, sponsored by the U.S. Department of Energy (DOE), and initiated in Fall 2003 at the Institute for Micromanufacturing (IfM), at Louisiana Tech University. The main objectives of this project, which have been successfully carried out, have been the development of a roadmap on nanotechnology for nuclear nonproliferation applications, and the implementation, through research and development, of an element of the roadmap. The efforts associated with the realization of a roadmap culminated in a July 2004 workshop for DOE and its partners, focusing on new technologies for i) detection of radiation and radioactive species, ii) collection, concentration, and detection of nuclear chemical species, and iii) detection of other signatures. Since the workshop, efforts towards the realization of an element of the roadmap have also been carried out at the IfM and highlighted in previous reports. The present report describes the work that has been accomplished in the final period of the grant, which has formally ended on September 29, 2006. This work has been on Multi-Channel Nanoparticle Scintillation Microdevice with Integrated Waveguides for Alpha, Beta, Gamma, and Neutron Detection. Details are provided in the following sections.

\section{Multi-Channel Nanoparticle Scintillation Microdevice with Integrated Waveguides for Alpha, Beta, Gamma, and Neutron Detection}

\section{Investigators}

Dr. Chester Wilson and Dr. Pedro Derosa are the lead investigators contributing to the realization

of this work. Specifically, Dr. Wilson has been responsible for the design and development of the device under consideration, and Dr. Derosa has led the theoretical and modeling investigation of the phenomena related to the given device.

\section{Main Objectives}

The main objectives of this effort are the development and prototyping of a small, sensitive, and low-cost multi-channel nanoparticle scintillation microdevice with integrated waveguides for 
alpha, beta, gamma, and neutron detection. This research effort has integrated experiments and simulation to determine the combination of process-specific materials for the achievement of optimum detection conditions.

\section{Rationale and Motivation}

The detection of gammas and neutrons is critical to homeland security applications because this type of radiation is emitted from fissionable bombs and difficult to shield. Traditional field detectors are Geiger counters, but these are large, typically shoe box sized devices. Although a MicroGeiger has been reported, it only detects beta-particles which are easily shielded [1]. A previous version of our nanoparticle loaded scintillators was reported, but it only detects beta and neutron radiation [2]. The device we have developed not only detects beta and neutron radiation but also discerns alpha, X-ray, and gamma radiation. As fissionable weapons grade material emits all five of these radiation species, including those hard to shield, this capability is central to a field detector that provides the exact composition of concealed nuclear material.

\section{Research Plan}

We have developed a glass microdevice with a new patterned nanoparticle composite resin that detects and discriminates all species of radiation emitted from fissionable bomb making materials. Tailored charge conversion nanoparticles doped into a fast electron scintillating resin produce different optical pulses specific to the radiation species. These pulses exit since the nanoparticles are appreciably smaller than the wavelength of light. The resins are integrated into a glass substrate where deep cavities are made using microsandblasting, forming independent optical paths leading to fiber-optic attachments. A separate, off the shelf Photo-Multiplier (PM) tube measures the light pulse. The different nanoparticles used convert differing radiation species into electrons through independent physical mechanisms, including charge conversion (alpha), secondary electron (beta), photoelectron (gamma/x-rays), and an on-chip thermonuclear fusion reaction (neutron) to evaluate the specific isotope radiation signature.

\section{Progress Report}

All radiation detecting elements are constructed by mixing charge conversion nanoparticles into a fast electron scintillating resin. $\mathrm{WO}_{3}, \mathrm{~Pb}_{3} \mathrm{O}_{4}$, and glass nanoparticles are used for beta, gamma/X-ray, and alpha detectors, respectively (Fig.1). Radiation impinging on the nanoparticles releases numerous lower energy electrons which scintillate the resin creating a light pulse. This pulse then exits the translucent detector since the diameter of the nanoparticles is smaller than the scattering threshold of light (Fig 1d). The scintillating resins are injected into a sandblasted glass microdevice; the glass is etched to provide cavities for the detectors, optical pathways, and fiber optic connectors (Fig. 2).

A cross sectional view of the detector without the silicone illustrates the design of the device to reduce optical cross talk between channels (Fig. 3). The final device contains four specific channels for alpha, beta, gamma/X-ray, and neutron detection (Figs. 4 and 5). Neutron detection is accomplished by doping the resin with $\mathrm{Gd}_{2} \mathrm{O}_{3}$ which releases an electron after a fusion reaction occurs (Fig. 6). 


\section{Experimental Results (Chester Wilson)}

The beta detector was tested using a ${ }^{36} \mathrm{Cl}$ source to compare its performance to traditional detectors, and the drop in count rates as the detectors distance from the source increases was found to be similar (Fig. 7). ${ }^{60} \mathrm{Co}$ emits both gamma and beta radiation; beta particles are easily shielded, gammas are not. Sheets of lead were used to shield the beta particles and test gamma/X-ray vs. beta-particle detector characteristics (Fig. 8). With no lead shielding, both betas and gammas are detected, as the lead thickness is increased, only gammas are detected.

Alpha detection was tested from a beam source (Fig. 9). After passing the high-flux-region, the count decreases as the detector is moved away from the source. The four different detectors use four different methods to convert four different types of radiation into electrons; as a consequence the measured pulses are characteristic to the radiation, allowing pulse height spectroscopy to be used (Fig. 10).

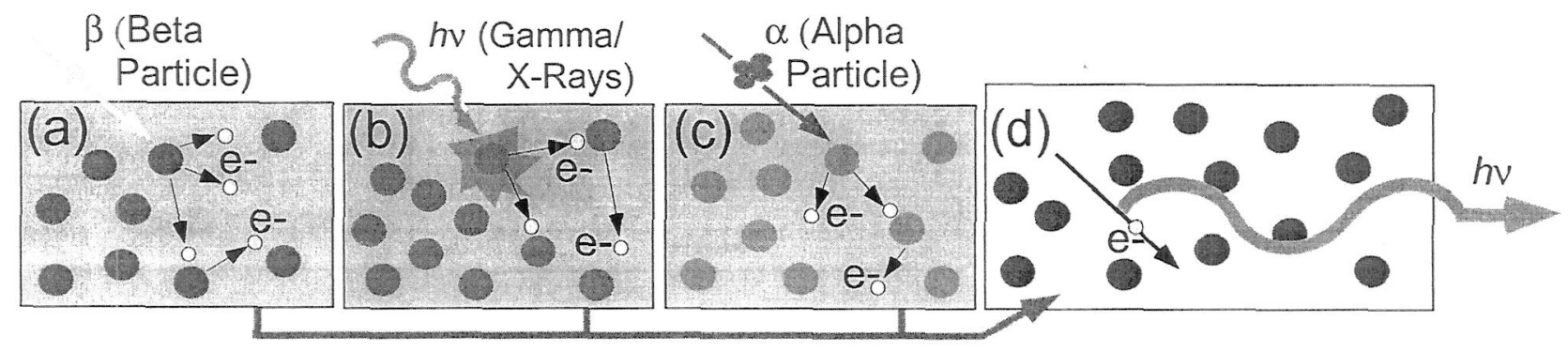

Fig. 1: Nanoparticle radiation scintillators; by doping fast electron scintillating resin with secondary electron metaloxide nanoparticles, the electrons become localized (a). Lead oxide nanoparticles convert impinging gammas/X-rays into photoelectrons (b), while glass nanoparticles convert alphas into electron cascades (c). The electrons are converted to light pulses by the scintillator, and are able to exit, as the nanoparticles are smaller than the scattering threshold of light (d).

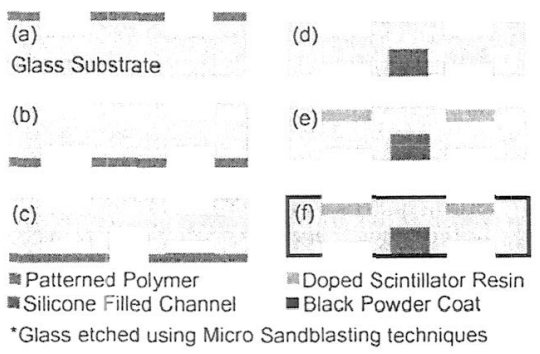

Fig. 2: Patterned polymer mask provides detector cavity (a). Second mask provides fiber optic insert (b). Third mask used to etch cross talk barrier (c). Injected silicone (d) and doped resin (e). Powder coat glass substrate (f).

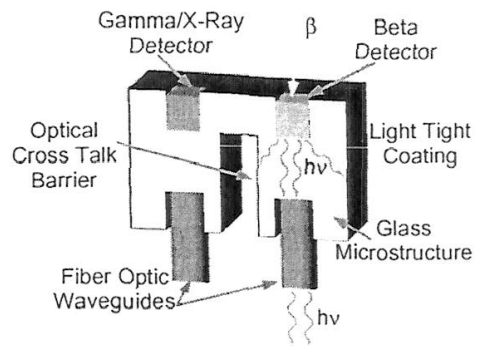

Fig. 3: Device is fabricated using microsandblasting; contains optical barriers, scintillator cavities, and fiber optic mounts patterned into the all glass structure, which is powder coated black.

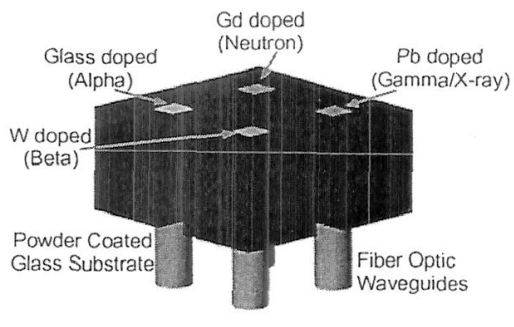

Fig. 4: Glass microdevice with four patterned radiation detection channels separated out for alpha (glass), beta (W), gamma/X-ray (Pb), and neutron (Gd) detection integrated with fiber optic waveguides. 


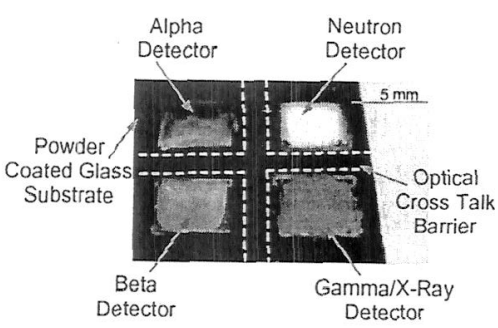

Fig. 5: Photo of four channel radiation detector shows independent beta, gamma/X-ray, neutron, and alpha particle channels. Outlined in yellow is the optical cross talk barrier with injected silicone contained on the bottom side of the detector.

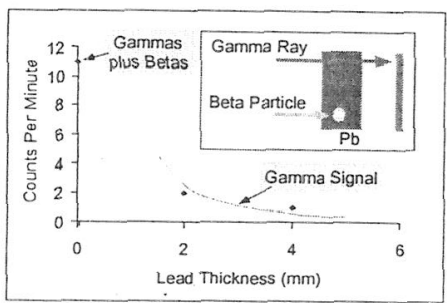

Fig. 8: ${ }^{60} \mathrm{Co}$ emits both gammas and betas so lead sheets are used to block betas in order to detect only gammas and demonstrate the difficulty in shielding gammas.
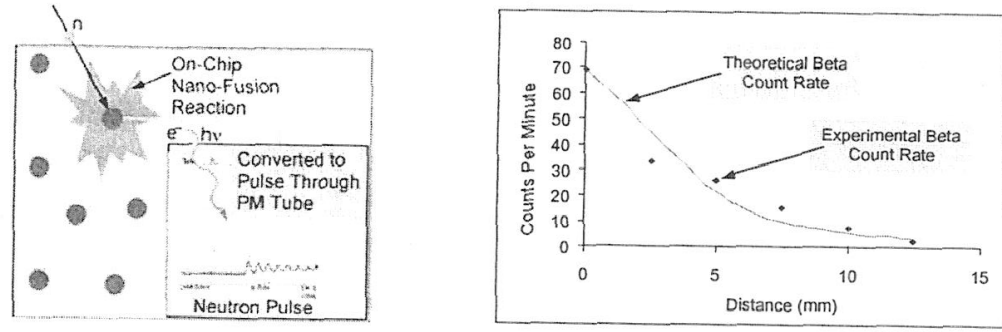

Fig. 6: $\mathrm{Gd}_{2} \mathrm{O}_{3}$ nanoparticles offer outstanding neutron-to-electron conversion, with a cross-section of 255,000 barns at thermal energies. Imbedded into the scintillator, they allow neutron detection when used with a pulse multiplier tube.

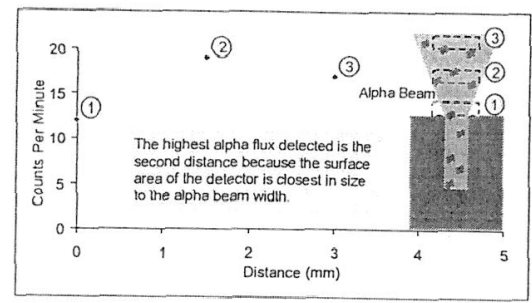

Fig. 9: ${ }^{241}$ Am emits an alpha beam with an increasing width to a certain limit. The detector converts the most alphas when the beam width is approximately the same size as the detector.
Fig. 7: Testing the beta detector with a ${ }^{36} \mathrm{CI}$ beta source for a function of counts per minute with distance as the variable compares to the theoretical beta count rate for various detectors on the market.

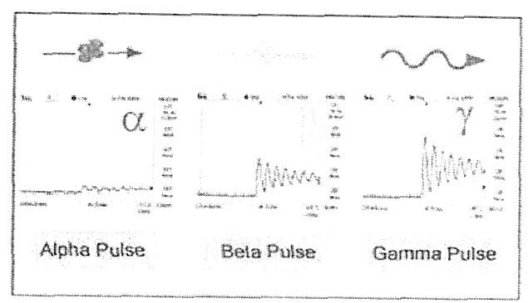

Fig. 10: Tailored resins use different conversion mechanisms producing varying PM tube outputs allowing for the capability of pulse height spectroscopy. 
Previous efforts to utilize Gd to detect neutrons, use it as a foil or a macroscopic block, a design that met with limited results; gadolinium is a metal, so it traps the electron. By utilizing gadolinium oxide as nanoparticles, the electrons can escape (Figure 15). Initial proof-of principle devices utilizing this technology have been constructed at the Institute for Micromanufacturing at Louisiana Tech University for initial testing with excellent results. Gadolinium oxide nanoparticles, which by themselves are opaque (Figure 16), have been mixed with fast-electron scintillating resin (Figure 17). This mixture has been spun into a thin film that can then be patterned into devices. A representative micro-scintillator made with this technique is shown in Figure 18.

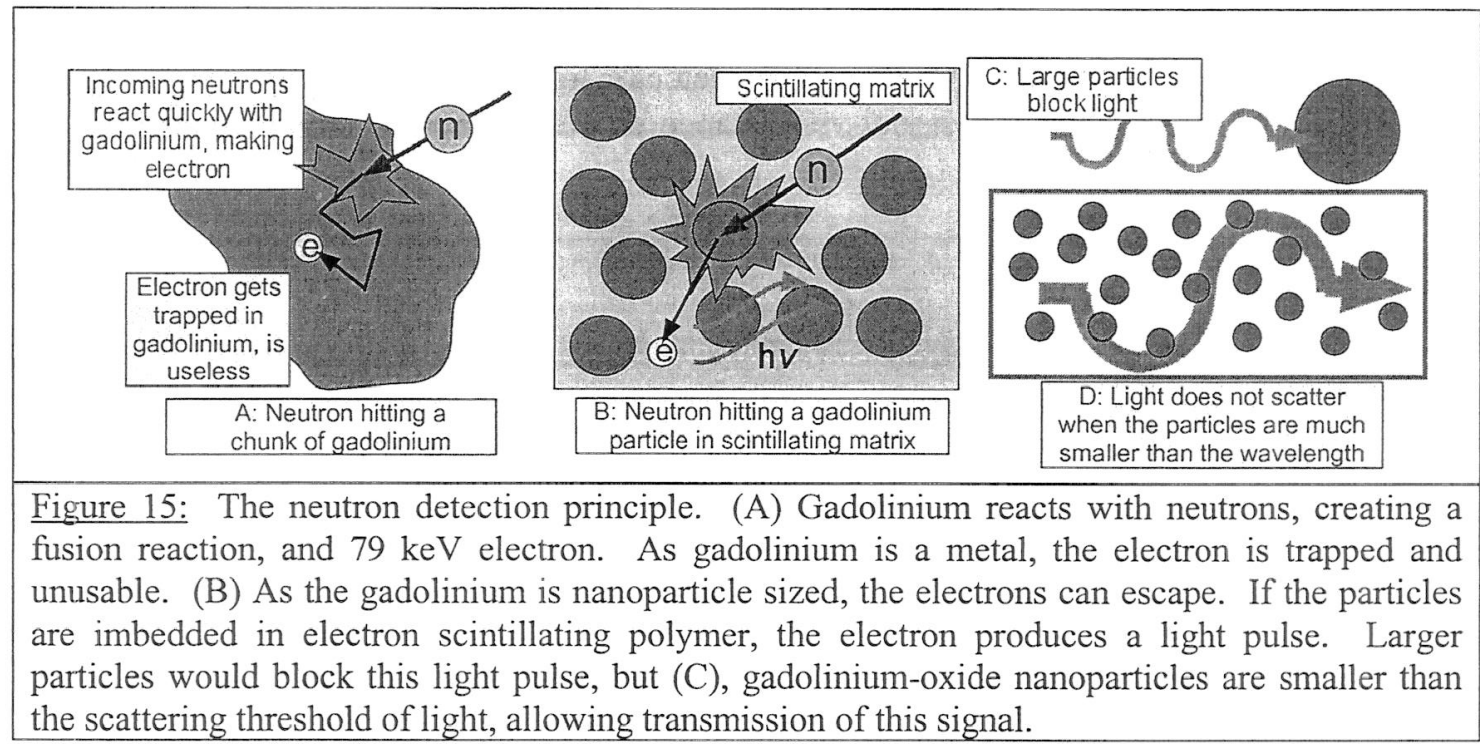

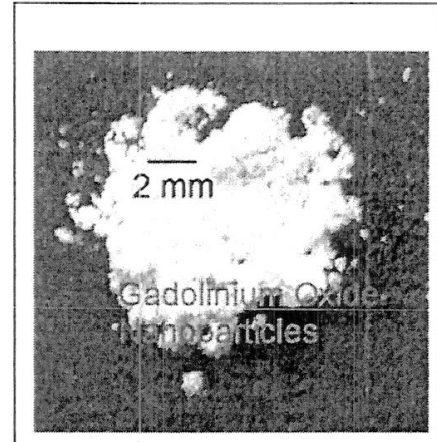

Figure 16: $\mathrm{Gd}_{2} \mathrm{O}_{3}$ nanoparticles offer excellent neutron-toelectron conversion, but by themselves are opaque.

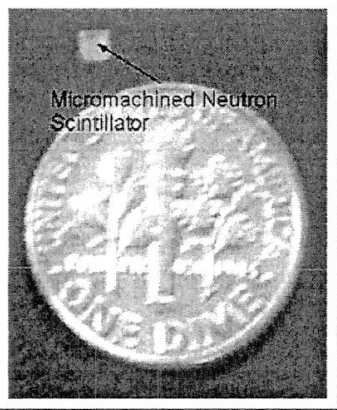

Figure 17: $\mathrm{Gd}_{2} \mathrm{O}_{3}$ nanoparticles/ scintillating resin can be manufactured into an easily patterned thin-film, allowing microdevice fabrication.

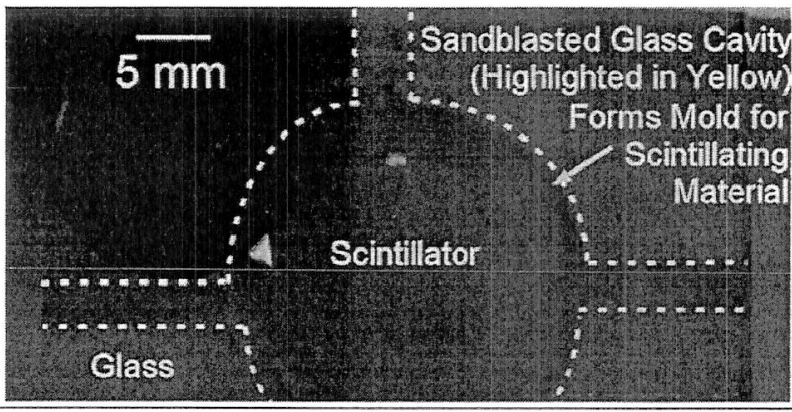

Figure 18: Transparency! The $\mathrm{Gd}_{2} \mathrm{O}_{3}$ nanoparticles are much smaller than dimensions which scatter visible light - so they will transmit the optical signal well. This scintillating material contains $30 \% \mathrm{Gd}_{2} \mathrm{O}_{3}$ by weight, and is 2 $\mathrm{mm}$ thick, but remains clear. Future devices will be patterned onto micromachined glass waveguides, allowing microdevices that can be directly connected to fiber optic bundles. 
This nanoparticle-loaded resin offers a number of ways to be constructed into useful smaller scale devices. A glass wafer was etched by sandblasting, and the cavity was used as a mold for the scintillating material. Since the gadolinium oxide nanoparticles are appreciably smaller than the wavelength of the scintillating light, there is negligible scattering of this light - the device in Figure 18 remains transparent despite the fact that the scintillating material is $2 \mathrm{~mm}$ thick, and is $30 \%$ gadolinium oxide. In future devices, the glass can be machined to become on-chip waveguides, with scintillators optimized for various parameters patterned onto the device. A fully packaged MEMS neutron scintillator array will be realized.

Optical transparency of the scintillating material was measured, and found to be high even with significant particle loading (Figure 20). The scintillator shown in Figure 18 was tested with an off-the-shelf Hamamatsu photomultiplier optical detector tube, and Am/Be neutron source. The device was found to have similar signal-vs.-distance characteristics as larger detectors (Figure 21).

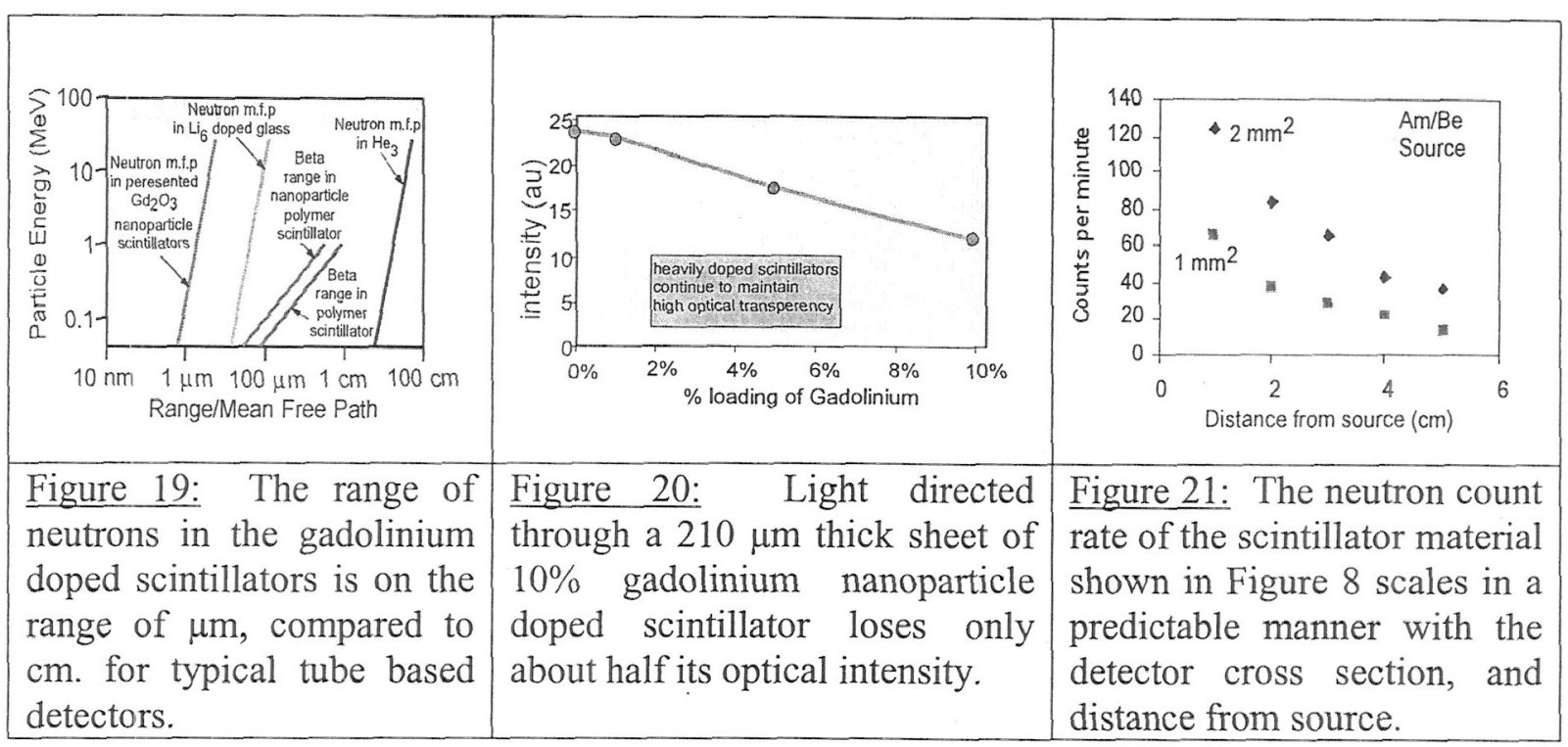

Integrated glass waveguides and a microfluidic channel have been constructed by masked sandblasting with micron-sized grit. Following fabrication, the beta-scintillating polymer is deposited (Figs. 22, 23). A drop of $\mathrm{P}_{32}$-doped water traversing the channel resulted in location dependent optical pulse rates measured from the waveguides (Figure 23). This allows submillimeter spatial imaging of beta-isotope tagged biomedical samples in lab-on-a-chip systems, by viewing differential signals. This scintillator material can be doped with charge conversion metal oxide nanoparticles, similarly to the neutron detection device. Metal oxide particles, upon bombardment by higher energy beta particles emit one or more lower energy electrons, which are better confined in the scintillator (Figure 22). Figures 26 a and b show characteristic pulses from an gadolinium oxide, and a tungsten oxide doped scintillator, respectively. 


\begin{tabular}{|c|c|c|}
\hline 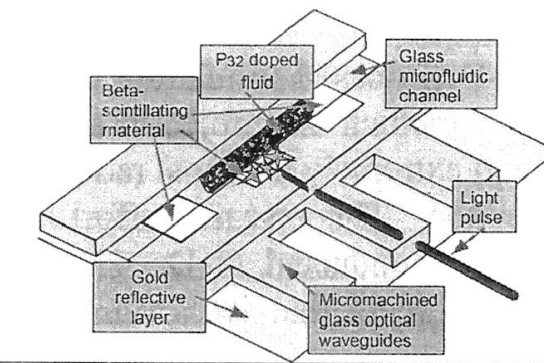 & & Normalized Distance Along Microchannel \\
\hline $\begin{array}{l}\text { Figure 22: A microfluidic } \\
\text { channel, and integrated } \\
\text { waveguide structure allows } \mathrm{P}_{32} \\
\text { doped fluid to pass over the } \\
\text { patterned beta particle } \\
\text { scintillators, and optical signal } \\
\text { measured from the waveguides. }\end{array}$ & $\begin{array}{l}\text { Figure 23: r } \\
\text { microsandblasted optical } \\
\text { waveguides (microchannel } \\
\text { removed for clarity). } \\
\text { Locally patterned beta } \\
\text { scintillators direct light } \\
\text { through the waveguides. }\end{array}$ & $\begin{array}{l}\text { Figure 24: Count rates per } \\
\text { minute vs. the normalized } \\
\text { distance of the drop of } \mathrm{P}_{32} \\
\text { doped water along the } \\
\text { waveguide. Count rates from } \\
\text { a waveguide are maximum as } \\
\text { the drop passes over the } \\
\text { corresponding detector. }\end{array}$ \\
\hline
\end{tabular}

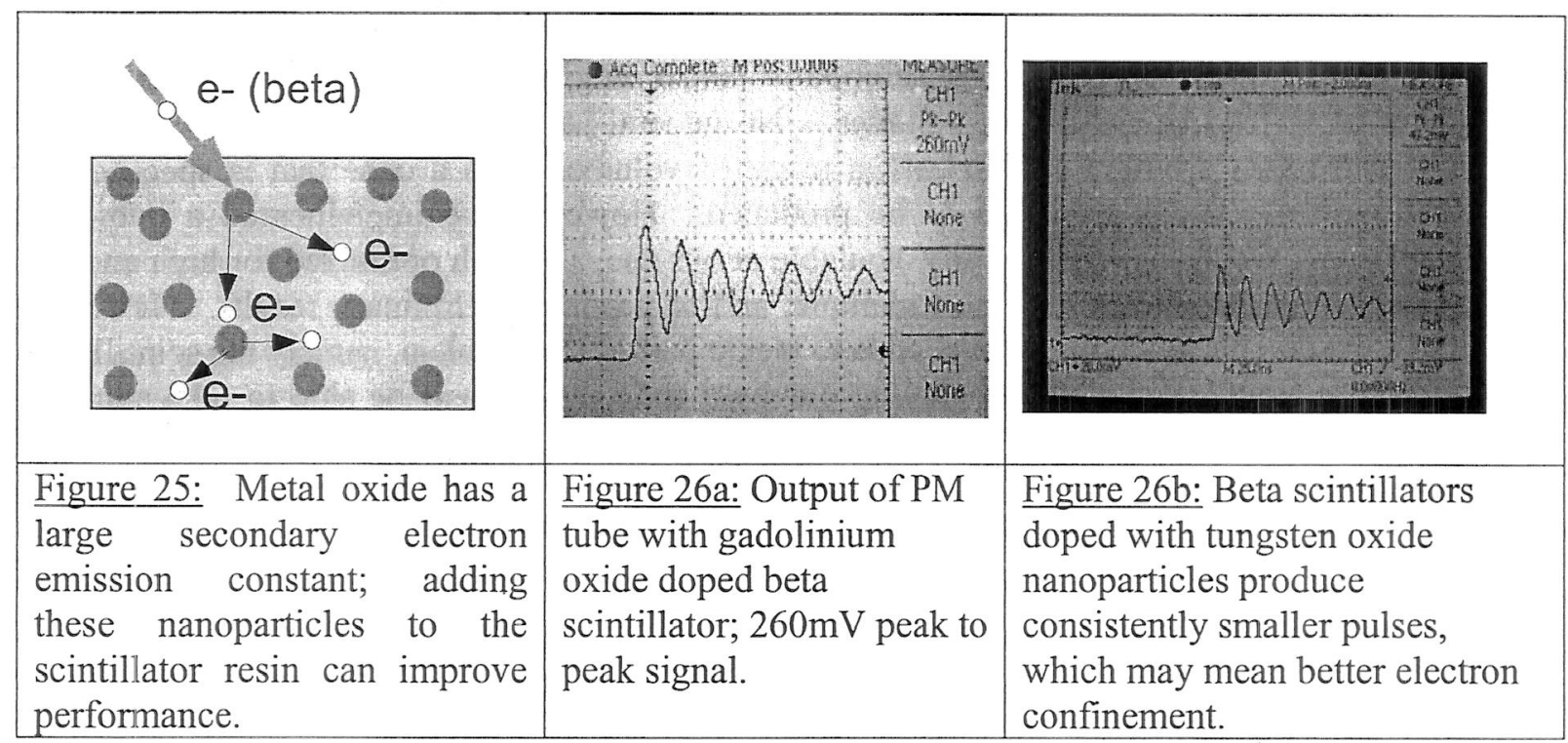

The integrated glass waveguides/nanoparticle scintillator technology will also be extended into gamma and X-ray detection. Nanoparticles with high photoemission, and lower photon range characteristics, such as $\mathrm{Pb}_{2} \mathrm{O}_{3}$ will be added to the scintillator matrix (Figure 25). These particles, combined with the secondary electron multiplying nanoparticles convert high energy photons into fast electrons, which the scintillator converts into light pulses. Initial scintillators have been constructed and doped with $\mathrm{Pb}_{2} \mathrm{O}_{3}$. These devices were tested with a cobalt gamma source, and they were found to produce large optical pulses (Figure 24). 


\section{Monte Carlo Simulation Results (Pedro Derosa)}

The main objective of this project is the development of a Monte-Carlo-based simulation model for radiation interaction with a detector device. The overall effort, of which this project is a part, consists of the design and development of small, very sensitive, and extremely low-cost radiation detectors, with excellent spatial resolution and particle discrimination. This research effort has been synergistically integrated into ongoing experimental research conducted in Dr. Chester Wilson's lab, to such an extent that we have recruited a Ph.D. student that is carrying out research in both labs. The detection technology we attempt to develop consists of nanoparticles embedded on a scintillating resin. The role of the nanoparticles is to convert the incoming radiation into electrons that will scintillate the resin. The essential advantage of using nanoparticles is their size is that they are expected to interact with the incoming radiation, since they are selected to be of a material displaying high cross-section towards radiation, but not greatly attenuating the electrons. produced in the interaction. Moreover, since they are smaller than the photon's wavelength, they will be virtually transparent to the photons produced in the scintillator.

From the simulation point of view, it is a formidable challenge to capture all the important aspects of these devices. Nanoparticles in the system will have diameters typically a million time smaller than the linear size of the scintillator matrix they are embedded in, which accounts to a factor of $10^{18}$ when comparing volumes. No general purpose computer code that we are aware of can retain accuracy within such a range of volumes, thus a code that is specifically designed for these problems needs to be produced. However, we implemented a two-step approach. First, we used a code readily available at our site, although optimized for high energy particles, to train our students in the technique, and to produce preliminary results. Next, we acquired a general purpose commercial code to tackle part of the problem, namely the scintillator matrix. Subsequently, we started producing our own code, which will be able to integrate the nanoparticles into a macroscopic material following a smart algorithm (the ghost nanoparticle) that is described below. The PI has hired a student from the Computational Analysis and Modeling Ph.D. program to assist in this task. There is also an M.S student working on this project.

The main tasks accomplished are as follows:

1. Training of students in radiation physics and preliminary results with GEANT3 simulator

2. Acquisition of MCNP5 simulator and training of students on its use

3. Study of optimum composition of scintillating matrix

4. Test of MCNP5 performance with high atomic weight material and small volumes

5. Development and implementation of a code to study interaction of radiation with nanoparticles (component of a more general code under development).

We started this project with the single objective of designing small, cheap, and selective neutron detectors motivated by the idea of their use in monitoring illegal activities for nonproliferation applications and homeland security. The detection of neutrons - in a location where no nuclear activity is expected - is beyond doubt an indication of illegal use of nuclear energy as neutrons are largely emitted by fissionable materials used in nuclear bombs, while only a very small number of other materials emit neutrons [3]. Experimental preliminary results have proved that 
devices like those proposed here are possible. We have planned this project convinced that with the support of computer simulation the design process can be optimized, not only reducing experimental efforts but also increasing our understanding of the observed processes.

As we became familiar with the device, it became obvious that there was no reason for limiting ourselves to neutron detection. In June 2005, the PI of this project and a group of other faculty from Louisiana Tech University and Grambling State University, partnered and produced a proposal to DoE, requesting $\$ 1.4 \mathrm{M}$ for the development of a sensing platform for neutron, beta, gamma, and $\mathrm{x}$-ray radiation. Support obtained for the project we are reporting here, gave us the opportunity to train students, produce preliminary results, and devise a viable plan for the design of a novel, nanotechnology-based radiation platform. Although we were not successful in getting funding for the DoE proposal, the comments of the reviewers were very encouraging, mentioning as a strong point the technical aspects of the proposal. On the whole, we believe that the reviewers' comments are a clear indication that we are going in the right direction. Based on these comments, we submitted another proposal to the department of homeland security last june 2006 and we are expecting a response at this moment.

\section{1- Monte Carlo Simulations Results with GEANT3}

The Monte Carlo technique (MC) is the most commonly used technique to study radiation interaction including production of secondary radiation. During a simulation, primary and secondary particles are followed until they are completely absorbed or until they leave the simulation area. The type of interaction and secondary products of those interactions are selected randomly with appropriate probability distributions. The predictions of this technique are the average of a large number of similar events [4] .

Our original plan was to use GEANT4 [5], the current version of the GEANT code, suitable to study the response of different parts of a material system to radiation. However, due to several problems in the implementation of the code that other faculty members in the Physics Program experienced, we decided to work with GEANT3 [6], the previous version of the code, which has been well-tested and is known to produce acceptable results. In addition, GEANT3 is written in FORTRAN, the language the PI of this project knows very well (GEANT4 is written in $\mathrm{C}++$ ).

\section{Selection, Installation, and Setup of the Monte Carlo Code}

Access to a computer cluster in the Physics Program at Louisiana Tech was granted, where the required CERN libraries are installed and GEANT3 is up and running. This step was only about setting up the account given to us to be able to run the code.

\section{Student Training}

Two students were recruited in the PI's research group and trained in the use of workstation running Linux OS and on the use of GEANT3. In a relatively short period of time, they learned how to work in a Linux environment interfacing from a PC, they learned the basis of radiation interaction with matter, and they became proficient in the use of the code.

Initial Setup of the Simulation 
To setup a simulation, the user needs to define all the parts of the experimental setup. Material composition, shapes, positions, sizes, etc. are setup by the user. GEANT3 can consider a number of elemental medium (materials) a particle can travel through in an experimental setup;

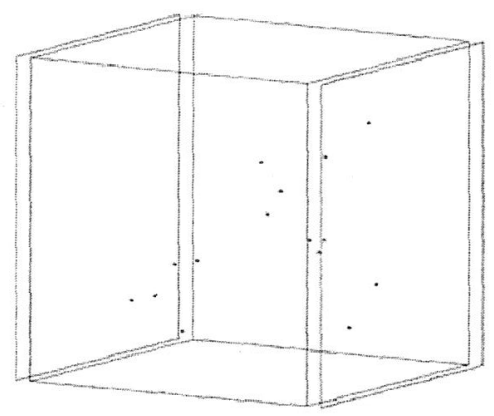

Figure 1: Blue cube is Bicron Scintillator, black dots are $\mathrm{Gd}_{2} \mathrm{O}_{3}$ nanoparticles. The purple squares are focal point detectors. composed materials, such as air, glass, etc. can be defined by the user in one of the input files. The geometry of the desired setup can be defined by combining pre-coded shapes, their dimensions are defined in an input file. If more complex geometries are needed, the user can include them by modifying the code. We have selected a box for our scintillator and spheres for the embedded $\mathrm{Gd}_{2} \mathrm{O}_{3}$ nanoparticles. In Figure 1 , a setup consisting on a few nanoparticles (black dots), embedded in bicron, a scintillator material (blue), is shown. The squares at each side of the cube are focal point detectors which are defined as made of "vacuum," and their objective is to keep track of the particles coming in and out of the box. Every particle that crosses the focal point detector is recorded together with its properties.

Test simulations were performed changing sizes, particles load, detector position, etc. This allowed us to get familiar with the use and capabilities of the software.

\section{Testing of the Code Precision}

GEANT3 was design to simulate complete detector settings for high energy particle experiments. Such detectors are macroscopic in size, some times several meters in diameter. Thus, we expected to have some problems for small systems such as nanoparticles, as this code was not designed with nanoparticles in mind. We carried out a simple experiment where $79-\mathrm{keV}$ electrons are made to interact with bicron slabs of different thicknesses between $12.5 \mu \mathrm{m}$ and 2 $\mathrm{cm}$. Theory predicts that the transmission probability decreases exponentially with the thickness of the material. Our results show the exponential decrease predicted by theory (Figure 2), but with two different slopes, one for low thicknesses, up to around $\sim 500 \mu \mathrm{m}$, and the other for thicknesses larger than $2 \mathrm{~mm}$. We believe that multiple interactions, more likely to occur in thick materials, are responsible for this. Probability of interaction and transmission probability do not necessarily follow the same trend, and electrons that suffer an interaction can still leave the material. In our simulation, some of them (those still traveling in the direction of the detector) will be counted as transmitted. Thus, for thicker materials, the transmission probability is expected to decrease at slower pace than the increase of the interaction probability. 


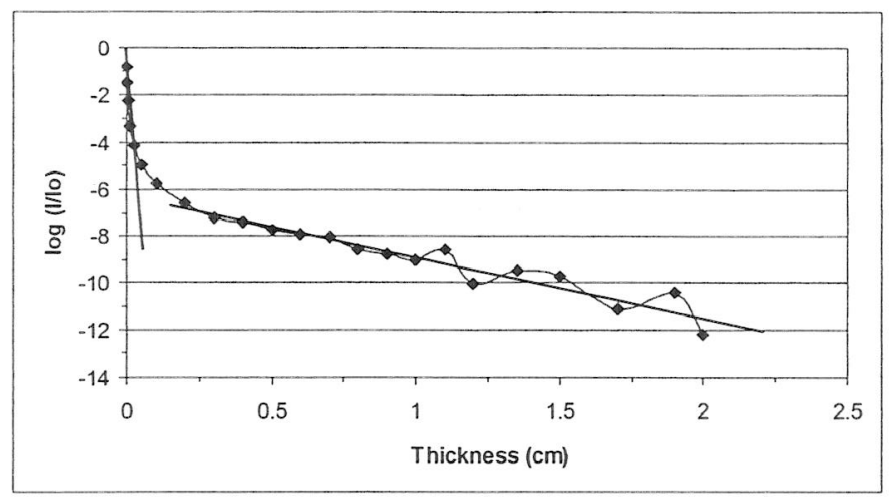

Figure 2: Transmission intensity of 79-kev electrons through a bicron slab vs. slab thickness. Overimposed lines are to guide the reader and do not correspond to a linear fitting. Oscillations at large thickness are due to numerical errors due to the small count of transmitted electrons.

Oscillations observed for large thickness are due to numerical errors due to the small number of electrons that are transmitted through the slab (see Table I). Table I shows the actual transmission data.

These results obtained provide us some confidence that numerical errors are not a problem down to tens of micrometers.

Interaction of Electrons with Bicron

The process we intend to simulate consists of two steps:

1. Incoming radiation interacts with nanoparticles. For example, when neutrons interact with $\mathrm{Gd}_{2} \mathrm{O}_{3}$ nanoparticles, electrons are generated (neutrons in $\mathrm{Gd}$ produce $79 \mathrm{keV}$ electrons).

2. Electrons interact with the scintillator. From this interaction, photons are produced.

We considered that the first step is to test the efficiency of the scintillator, and thus we considered $79 \mathrm{keV}$ electrons as primary radiation, and observed the production of photons in the scintillator. One of the main targets of this research is the production of small size detectors (millimeter and sub-millimeter). Thus, we studied the production of photons as a function of the scintillator thickness. Figure 3 illustrates the photon production for three values of thicknesses $20 \mathrm{~cm}, 2 \mathrm{~cm}$ and $2 \mathrm{~mm}$. 


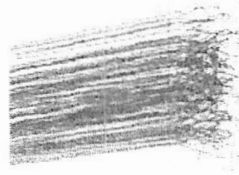

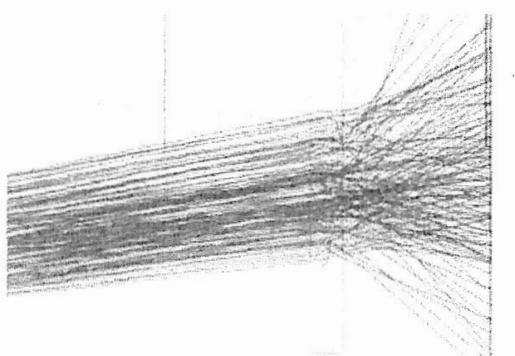

b)

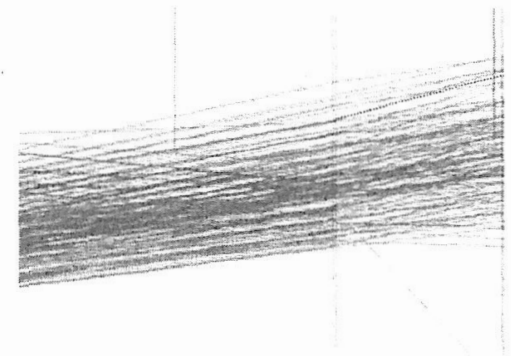

c)

Figure 3: $79 \mathrm{keV}$ electrons (red lines) interact with a scintillator material (blue box) of a) $20 \mathrm{~cm}, \mathrm{~b}) 2 \mathrm{~cm}$, and c) 2 $\mathrm{mm}$ thickness. As a result, photons are generated (dotted blue lines). The photon trajectory has been highlighted in c) to allow a clearer visualization.

Table I: Number of photons produced in a bicron slab of different thickness when irradiated with $79 \mathrm{keV}$ and $10 \mathrm{keV}$ electrons.

\begin{tabular}{|c|c|c|}
\hline $\begin{array}{c}\text { Slab thickness } \\
(\mathbf{c m})\end{array}$ & $\begin{array}{c}\text { Number of } \\
\text { Photon for } \\
\text { 79keV Electrons }\end{array}$ & $\begin{array}{c}\text { Number of } \\
\text { Photon for } \\
\text { 10keV Electrons }\end{array}$ \\
\hline 20 & 31227 & 7019 \\
\hline 2 & 37202 & 13436 \\
\hline 0.2 & 3955 & 2950 \\
\hline 0.02 & 373 & 294 \\
\hline
\end{tabular}

In these simulations, we observed the effect of downsizing the detector volume, the efficiency is dramatically reduced. With the objective of increasing the efficiency, an alternative in the design consists of adding a second type of nanoparticles to capture fast $(79 \mathrm{keV})$ electrons and produce several lower energy electrons. To study the effect of this alternative, we repeated the simulation with $10 \mathrm{keV}$ electrons and compared the efficiency. The results are shown in Figure 4.

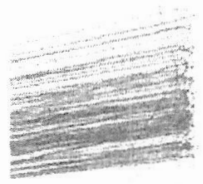

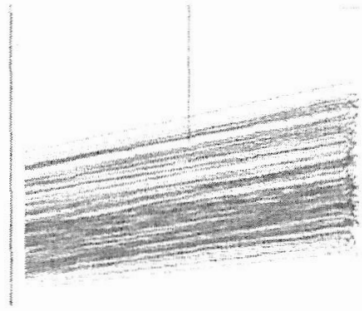

b)

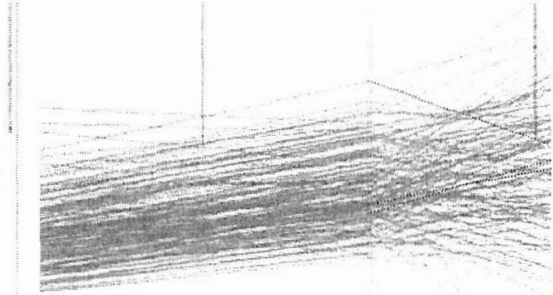

c)

Figure 4: $10 \mathrm{keV}$ electrons (red lines) interact with a scintillator material (blue box) of a) $20 \mathrm{~cm}$, b) $2 \mathrm{~cm}$, and c) 2 $\mathrm{mm}$ thickness. As a result, photons are generated (dotted blue lines). The photon trajectory has been highlighted in c) to allow a clearer visualization. 
Table I shows comparative results for 4 different slab sizes. In all cases, even for the $200 \mu \mathrm{m}$ case, $79 \mathrm{keV}$ electrons seem to be more efficient in producing photons than the $10 \mathrm{keV}$ electrons. It may however be noted that for the $2 \mathrm{~mm}$ and $200 \mu \mathrm{m}$ slabs, the photon intensity for $10 \mathrm{keV}$ electrons is $75 \%$, while for $79 \mathrm{keV}$ the efficiency is $78 \%$. This result means that even if only two, $10 \mathrm{keV}$ electrons are generated out of each $79 \mathrm{keV}$ electrons, an improvement of $\sim 100 \%$ will be observed in the photon intensity.

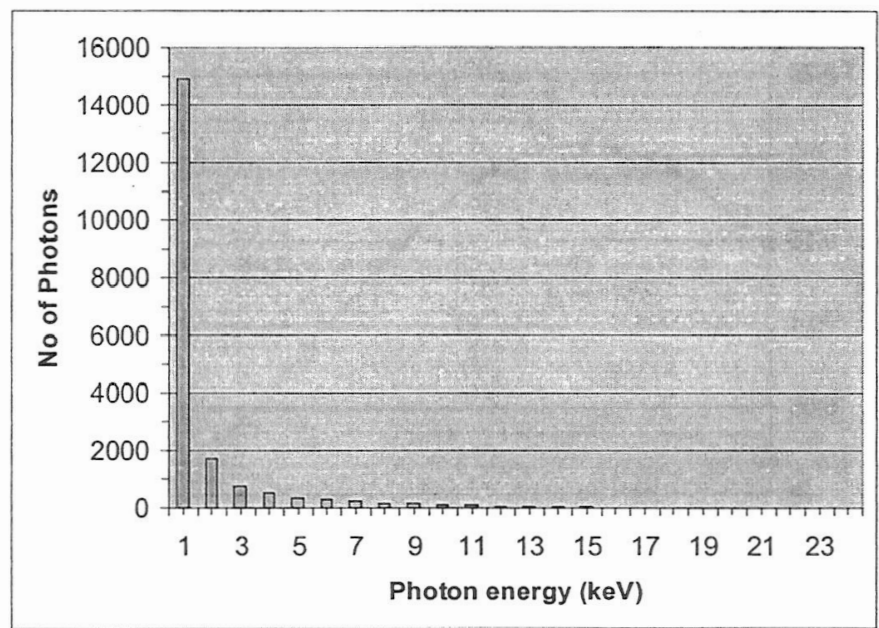

Figure 5: Photon spectra out of a bicron slab when irradiated with $79 \mathrm{keV}$ electrons.

\section{Study of the Effect of Glass Support}

The results described above do not seem to be in agreement with the experiment that predicts very high photon intensity. To test the reasons for such a high photon intensity, we worked on the hypothesis that in the experiment the support (boron silicate), on top of which the scintillator was deposited, may be either producing photons of its own, or backscattering electrons which now have another chance to interact with the scintillator. Figure 6 illustrates results comparing the generation of photons and backscattered electrons when a boron-silicate glass is positioned behind the scintillator. A larger dispersion of electrons (red solid lines) is observed due to the presence of glass (Figure 6a). However, no significant increase in the number of generated photons is observed. It is still not clear what the reason of the high intensity in the experiment or the low intensity in the simulation is. Studies are still carried out to understand this issue.

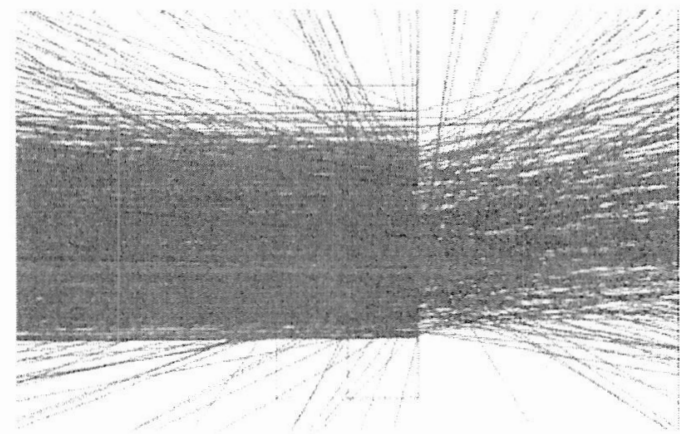

a)

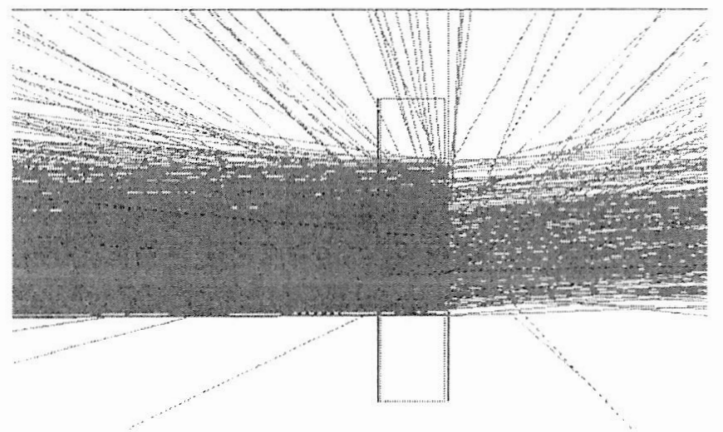

b)

Figure 6: $79 \mathrm{keV}$ electrons (red lines) interact with a) a $2 \mathrm{~mm}$ thick scintillator slab (blue box) mounted on top of a $2 \mathrm{~mm}$ glass (purple box). b) Only a $2 \mathrm{~mm}$ think scintillator slab. 


\section{The Electron Source}

The electron source in the experiment is not monochromatic, but it has a distribution given in Figure 7. During the simulation, different energies are selected and a number of electrons, proportional to the intensity at that energy are considered. The result for the entire spectrum is a superposition of the results at each energy level.

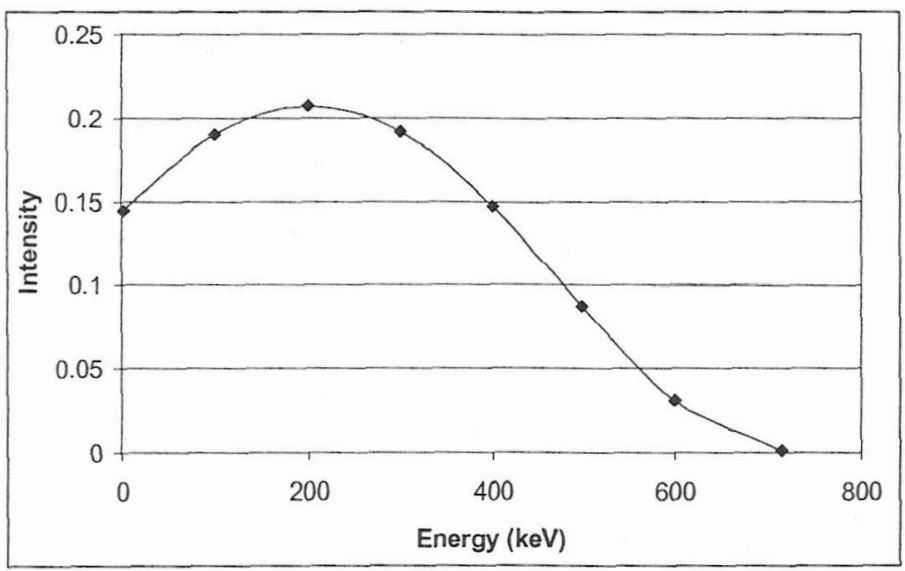

Figure 7: Electron spectrum generated in the simulation resembling that of the experimental electron source

Table 1I: Number of photons produced by electrons in a bicron slab as a function of its thickness.

\begin{tabular}{|c|c|}
\hline $\begin{array}{c}\text { Slab thickness } \\
(\mathbf{c m})\end{array}$ & $\begin{array}{c}\text { Number of } \\
\text { photons }\end{array}$ \\
\hline 0.25 & 1028 \\
\hline 0.5 & 4303 \\
\hline 1 & 7571 \\
\hline
\end{tabular}

With the electron source described in Figure 7, simulations were carried out where bicron slabs with three different thicknesses, supported on a $500 \mu \mathrm{m}$ glass, are irradiated with electrons. Table II shows the number of photon generated in each case.

\section{2- Acquisition of MCNP5 and its Use}

GEANT3 is a set of libraries optimized for the simulation of a high energy particle detection setup. It also works for other purposes, but it requires the programming of any new application, making its use complicated and time-consuming. In the preliminary calculations described above, code designed by members of the Physics Program was used. It was very important for the training of our students who, after a period of working with GEANT3, are able to understand the process of interaction of radiation with matter. 
At this point, the two students assigned to this project were reassigned; one of them was assigned to work with MCNP5 in the optimization of the scintillating materials, while the other was assigned to the development of a code able to implement the interaction with nanoparticles.

\section{Acquisition of MCNP5}

MCNP5, a code developed by the Oak Ridge National Laboratory (ORNL), is a general purpose Monte Carlo code to study interaction of radiation, with matter of particular specialization in neutron interaction. Due to the potential applications of a code like this, in activities that can risk US homeland security, ORNL conducts a background check on all potential users. We thus have chosen a student who is an American citizen to be in charge of the use of this code and applied for clearance for him. A license was granted to him for the use of this program.

\section{Student Training in the Use of MCNP5}

Due to the previous experience in the modeling of radiation detectors using GEANT3, it was relatively simple for this student to get acquainted with the new software. He only needed to get used to the new format of input and output files, and he did so by studying the response of different materials to incident radiation.

\section{3- Study of Optimum Composition of Scintillating Matrix (MCNP5)}

As described above, the process of radiation detection happens in two stages:

- Conversion of incident radiation into electrons

- Conversion of electrons into photons.

The first stage occurs in the nanoparticle. It is then important to select the nanoparticle material and size to optimize the interaction with the incoming radiation at the same time that the interaction of electrons with nanoparticle is reduced to a minimum to allow the generated electrons to exit the nanoparticle. The second stage happens in the scintillator.

The type and size of nanoparticle depends on the radiation to detect its energy. However, regardless of the nature of the incident radiation, the product of its interaction with the nanoparticles are electrons, and thus it is very important to study the response of the scintillator to electrons, particularly the production of photons, and optimize the scintillator composition to make the process more efficient.

Figure 8 shows the response to beta radiation (electrons) of a scintillator material compared to pure carbon and to water as a function of the electron energy. 


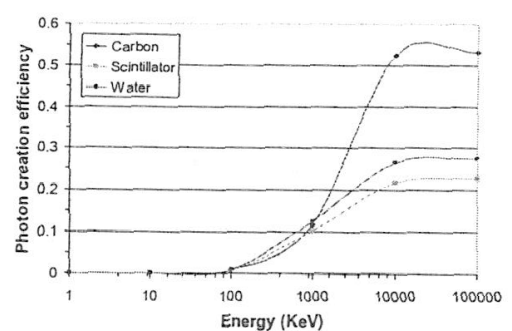

a)

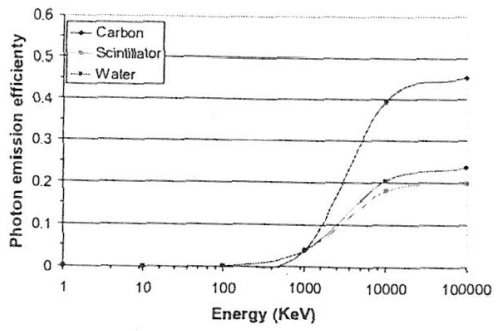

b)

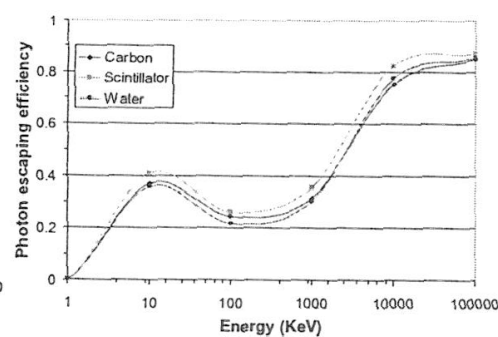

c)

Figure 8: Comparative response to beta radiation of Scintillator, Carbon, and Water as a function of the electron energy. a) Photon creation (photons created per incident electron), b) photon transmission (photons leaving the scintillator per incident electron), c) photon transmission efficiency (photon leaving the slab per each photon created) for a $1 \mathrm{~cm}$ slab. Scintillator's stoichiometry is $\mathrm{CH}_{1.1}$

Carbon shows to be twice as efficient in the creation of photons as bicron which shows a similar behavior to that of water (Figure 8a). This remains true when considering the transmission of those photons (Figure 8b). It may be noticed that all materials show a similar photon absorption behavior and also that the three materials show high absorption of photons created with electrons of around $100 \mathrm{keV}$ impinge the sample. In the previous case only $25 \%$ of the photons created leave the sample. This could be a problem when working with neutron detectors. Neutronelectron conversion is done by $\mathrm{Gd}$ nanoparticles that produce $79 \mathrm{keV}$ electrons for which reabsorption is high. It may be noticed that photons created with $10 \mathrm{keV}$ electrons are less efficiently absorbed than $79 \mathrm{keV}$ electrons. Adding a second type of nanoparticles, capable of reducing the incoming electrons energy to $\sim 10 \mathrm{keV}$, will improve detection efficiencies.

Figure 9, shows the results for a $0.5 \mathrm{~mm}$ slab. Reducing the slab thickness, as expected dramatically reduces the efficiency; however, it does not greatly affects the photon transmission efficiency thus allowing for the reduction in the device size.

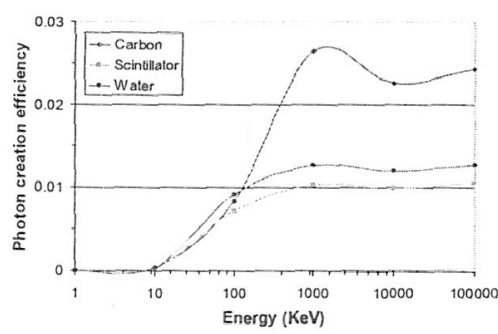

a)

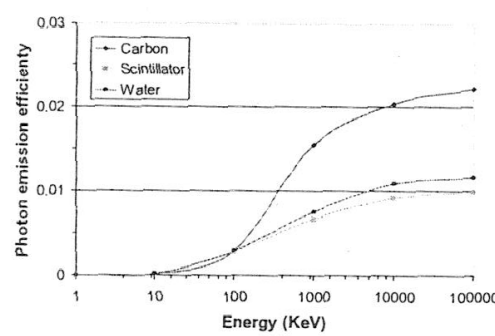

b)

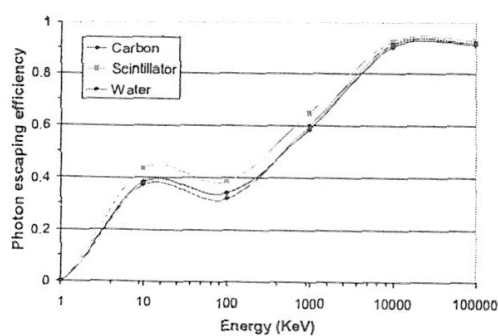

c)

Figure 9: Comparative response to beta radiation of Scintillator, Carbon, and Water. a) Photon creation (photons created per incident electron), b) photon transmission (photons leaving the scintillator per incident electron), c) photon transmission efficiency (photon leaving the slab per each photon created) for a $0.5 \mathrm{~mm}$ slab. Scintillator's stoichiometry is $\mathrm{CH}_{1.1}$

In the calculations described in Figures 8 and 9 , the density of the materials corresponds to the natural material, thus $\mathrm{C}$ density was $2.25 \mathrm{~g} / \mathrm{cm}^{3}, \mathrm{H}_{2} \mathrm{O}$ density was $1 \mathrm{~g} / \mathrm{cm}^{3}$, while for the scintillator the density was $1.03 \mathrm{~g} / \mathrm{cm}^{3}$. The higher efficiency of $\mathrm{C}$ is then understandable due to its higher density. In order to assess the importance of the material itself, comparative 
simulations between different materials where the density was set to $1 \mathrm{~g} / \mathrm{cm}^{3}$ for all of them, were performed.

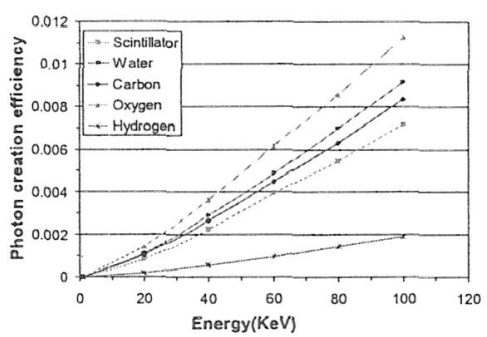

a)

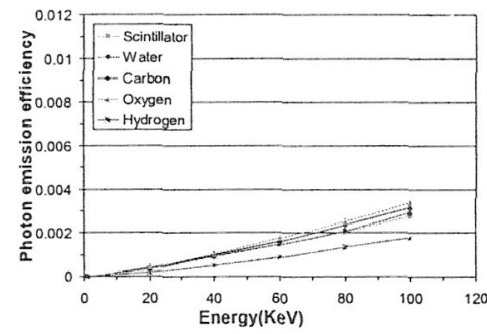

b)

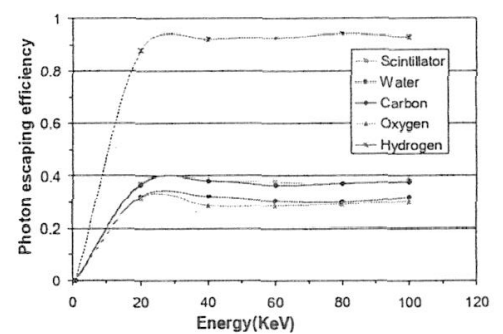

c)

Figure 10: Comparative response to beta radiation of Scintillator, Water and their constituent elements, C, O, and H. a) Photon creation (photons created per incident electron), b) photon transmission (photons leaving the scintillator per incident electron), c) photon transmission efficiency (photon leaving the slab per each photon created) for a $0.5 \mathrm{~mm}$ slab. Scintillator's stoichiometry is $\mathrm{CH}_{1.1}$

Figure 10 shows the response of scintillator and water compared to their constituent elements when all have the same density. Notice that $\mathrm{C}$ is only slightly better than the scintillator while water is the best. Also notice that the $\mathrm{H}$ performance in creating photons is very poor thus explaining the improved performance of $\mathrm{C}$ over scintillator that also has $\mathrm{H}$. Notably, $\mathrm{H}_{2} \mathrm{O}$ is better than both $\mathrm{C}$ and the scintillator, implying a good performance of $\mathrm{O}$ that shows to be the best in electron creation and transmission. For $\mathrm{H}$ the rate of photon escaping to photon created is by far the best, however due to its poor photon creation efficiency, overall $\mathrm{H}$ performs poorly. From this study, we have recommended the use of materials with less $\mathrm{H}$ and more $\mathrm{O}$. This was later confirmed by the simulation (Figure 11).

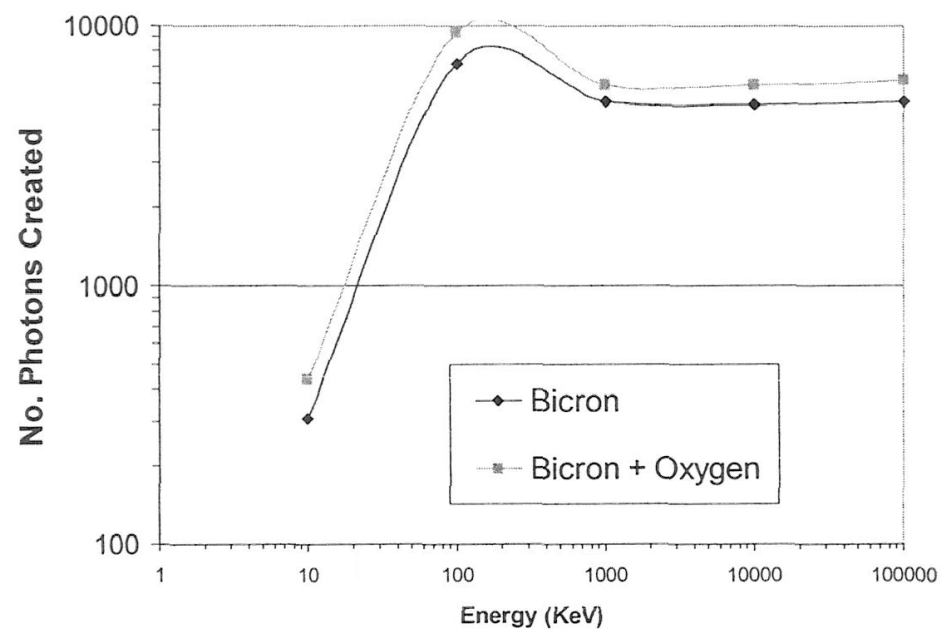

Figure 11: Photons created in bicron vs. photon created in bicron with extra oxygen. Photon creation is improved when more oxygen is added. 


\section{4- Test of MCNP5 Performance with High Atomic Weight Material and Small Volumes}

The performance of MCNP5 when dealing with high atomic weight materials was also studied. Results were not quite satisfactory regarding the interaction of neutrons with $\mathrm{Gd}$ and it is believed that some of the interactions, important to our problem, are not being correctly treated. We observed an alarming low rate of electrons produced in $\mathrm{Gd}$ following the interaction with neutrons, which is comparable, for instance, to boron. It is well known that the absorption crosssection for $\mathrm{Gd}$ is several orders of magnitude larger than that for boron and that $\sim 39 \%$ of the absorption interactions end up in an electron emission. Thus, these results are certainly incorrect. We determined that the beta emission following an absorption event it is not considered by default in MCNP5, thus explaining the low rate of electrons produced. We are currently trying to communicate with the developers, and the PI participated in a meeting at ORNL last month and made attempts to contact personnel related to this code. A person has been identified and contacted, and we expect he will lead us to the appropriate person to help with this issue.

Below we present some of the results obtained for electrons and photons that are considered more reliable.

\section{Electron-Electron Conversion}

We first studied the response of different materials to beta radiation namely $\mathrm{Gd}, \mathrm{Pb}$, and $\mathrm{W}$. The results are provided in Figure 12, showing the electron transmission intensity versus energy. Of these materials, $\mathrm{W}$ is the most efficient in its interaction with electrons, and thus $\mathrm{W}$ is a candidate for nanoparticle-based beta detectors.

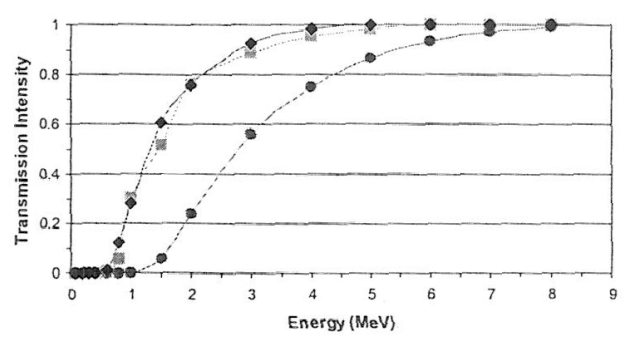

Figure 12: Electron Transmission through different materials

$\mathrm{Gd}$ and $\mathrm{Pb}$, although with similar efficiency, are in a different class with respect to $\mathrm{W}$.

\section{Photon-Electron Interaction}

The response of $\mathrm{Pb}, \mathrm{Gd}$, and $\mathrm{B}$ was comparatively studied. It was determined that $\mathrm{Pb}$ shows the best conversion rate as shown in figure 13. In addition, $\mathrm{Pb}$ shows the best absorption efficiency for photons that making it the best candidate for photon conversion (figure 14)

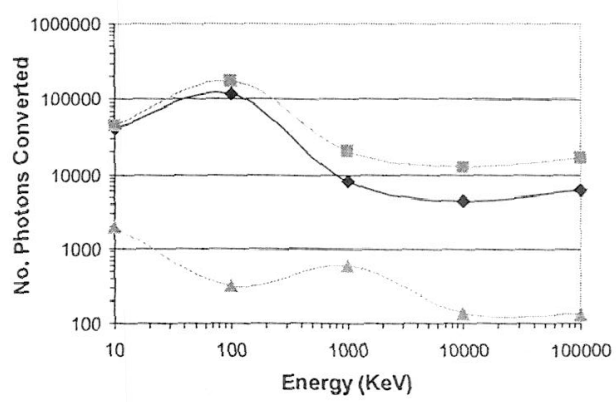

Figure 13: Photon-electron conversion in different materials 

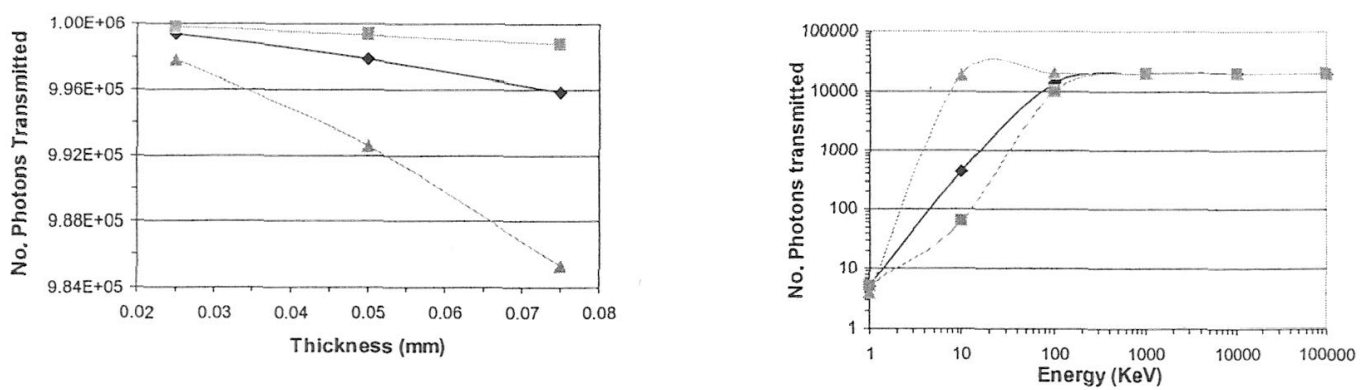

Figure 14: Efficiency of photon absorption as function of a) thickness b) energy. Pb shows the best absorption properties thus making $\mathrm{Pb}$ the best candidate for photon-electron conversion

Thus $\mathrm{Pb}$ is the best photon absorber and the best photon-electron converter, thus being them candidate for photon-electron conversion.

\section{5- Development and Implementation of a Code to Study Interaction of Radiation with Nanoparticles (component of a more general code under development)}

Due to the problems described in previous sections, including accurate treatment of a system composed of macroscopic and nanoscopic components and the issues with MCNP5, it was decided that an in-house code will be created. MCNP5 will still be used to optimize the matrix where nanoparticles will be embedded and to provide test cases for the code.

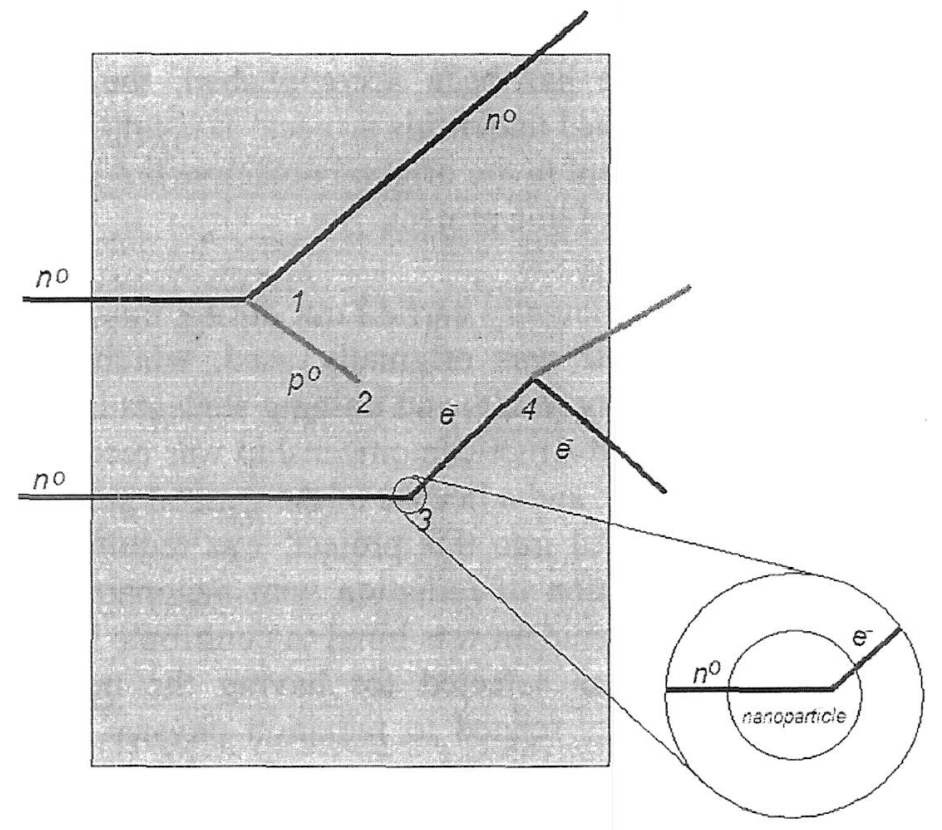

Figure 15: Schematic of the interaction of the "ghost nanoparticle" approach. Two neutrons are considered (black track), and randomly the first neutrons is considered to suffer a scattering while the second is consider to collide with a nanoparticle. A different routine studies the interaction with the nanoparticle and returns the control to the main program to track the product of the interaction of the neutron and the nanoparticle. A red track represents photons and blue tracks electrons.
In this section the general algorithm will be described. The approach to be followed was called "the ghost nanoparticle". The idea is simply to consider the interaction of an incident (or a secondary) particle with a nanoparticle as one of the possible events the incident particle can suffer together with absorption, elastic scattering, inelastic scattering, etc. In other words, the material and the incoming radiation it will interact with will have no nanoparticle explicitly included therein. However a possible "event", collision with a nanoparticle, will be included, and the probability for this "event" to occur will be the ratio between the area covered by the nanoparticles and the area of the slab the nanoparticle are immersed in. The algorithm is schematically 
represented in Figure 15 considering neutrons and incident particles. Two neutrons are represented in this figure. The first one interacts at point 1 with a scattering event, and as a results, a photon $\left(P^{\circ}\right)$ is produced that is absorbed at 2 , while the neutron after the scattering leaves the sample. The second neutron suffers a collision with a nanoparticle. This was decided via the generation of a random number. When this happens, the control is transferred to a subroutine that tracks the neutron inside the nanoparticle, resulting in the generation of an electron that suffers a scattering at 4 , producing a photon that leaves the sample. The electron itself leaves the sample after that interaction.

This approach has several advantages, on one hand nanoscopic and macroscopic objects in the same are not present at the same time in the same simulation box (nanoparticles are treated separately in a routine developed for that effect), both sizes can then be treated with equivalent precision. The second advantage is that there is no need to have a large number of volumes declared in the input file; nanoparticles are not formally treated as an object but as an interaction, thus nanoparticles are treated one at a time.

The section of the program dealing with nanoparticles is almost complete at this point. To speed up this development, a new student in the Ph.D. in Computational Analysis and Modeling program was recently hired. He was selected for having the needed computation skills and previous experience in some topics related to radiation physics.

\section{Overall Computational Efforts}

The main objective of this project has been the implementation of a simulation technique to complement the ongoing experimental work in the design of radiation detectors, and the training of students in these techniques. $90 \%$ of this objective has been accomplished; the only remaining activity is to finish the computational code designed under this project. It is important to mention that the development of the in-house code, was not in the original objective but it was determined to be absolutely necessary during the execution of this project.

Activities were planned in such a way that they can be efficiently carried out during this work minimizing any delays. Thus, a less-than-adequate code was originally used, which was however readily available, and allowed gathering preliminary results and training students in this type of simulations. In the mean time, a Ph.D. student with American citizenship was recruited that joined both, the experimental and the simulation efforts, and a license of the general purpose code MCNP5 was requested. An MS student, also recruited into this project, was required to initiate the programming of the code to study the interaction of radiation with nanoparticles. Recently a new Ph.D. in Computer Analysis and Modeling student was hired to contribute in the design and development of the in-house code. He was selected for having the needed computation skills and previous experience in some topics related to radiation physics. This student is now the main person responsible with the development of the new code, while the MS student, with more experience in this area, will assist with the more technical aspects.

The main findings are summarized below:

1) The efficiency of the device is predicted to increase if a second type of particle converting high energy electrons into more less energetic electrons are used. 
2) Scintillator efficiency is expected to increase with a larger concentration of oxygen in the scintillator resin.

3) Tungsten was determined to be the best choice for beta detection due to its superior electronelectron conversion properties.

4) $\mathrm{Pb}$ was determined to be the best choice for beta detection due to its superior photon-electron conversion properties.

5) To efficiently study nanoparticle doped scintillator an in-house code is needed and this is a work in progress.

\section{References}

1. C. Wilson, C. Eun, Y. Gianchandani, "D-MicroGeiger: A Microfabricated Beta-Particle Detector with Dual Cavities for Energy Spectroscopy", IEEE MEMS 2005, Jan. 2005, pp. 622-625.

2. R. Dasaka, et al., "Micromachined Scintillation Devices with Charge Conversion Nanoparticles for Neutron and Beta Particle Detection", $\mu$ TAS 2005.

3. Knoll, G., Radiation detection and measurement. $3^{\text {rd }}$ ed. 2000: John Wiley \& Sons, Inc. 802.

4. Allen, M.P. and D.J. Tildesley, Computer simulation of liquids. 1990, Oxford: Clarendon Press.

5. GEANT4, user manual CERN. 1998: <wwwasd.web.cern.ch/wwwasd/geant/>.

6. GEANT3, User manual, C.a.N.D. Application Software Group, Editor. 1993, CERN: Geneva, Switzerland. 
